\title{
STEROID REGULATION OF NEURONAL DEATH IN THE MOTH NERVOUS SYSTEM ${ }^{1}$
}

\author{
JAMES W. TRUMAN AND LAWRENCE M. SCHWARTZ ${ }^{2}$ \\ Department of Zoology, University of Washington, Seattle, Washington 98195
}

Received May 23, 1983; Revised August 15, 1983; Accepted August 16, 1983

\begin{abstract}
The emergence of the adult Manduca sexta moth is followed by the programmed degeneration of about $50 \%$ of the insect's abdominal interneurons and motoneurons. Neurons in implanted ganglia undergo degeneration in concert with neurons of the host, thereby indicating that a hormonal cue is important. The neuronal death follows the normal decline in the levels of the steroid hormones, ecdysteroids, that occurs at the end of metamorphosis. Manipulations that cause a precocious ecdysteroid decline result in an early death of the neurons. Also, prolongation of the ecdysteroid titer extend the life of the neurons. By properly timed steroid application it was possible to dissociate the neuronal degeneration from the behavioral events and muscle degeneration that also occur at emergence. Also the stereotyped sequence of death among identified motoneurons could be stopped at various points in the sequence by the carefully timed application of steroid. It was concluded that the signal for neuronal death was the withdrawal of ecdysteroids at the end of metamorphosis and that the death was probably a direct response of the individual neurons to the hormone withdrawal.
\end{abstract}

Programmed cell death is a ubiquitous feature of developing nervous systems. This cell degeneration occurs in a number of contexts. In many regions of the CNS, neurons appear to be overproduced, and then interactions with pre- or postsynaptic cells determine which neurons will live and which will die (Hamburger and Oppenheim, 1982). In other cases a particular neuron may have only a transient function during the life of the organism, and after that function is completed the cell then degenerates. This latter phenomenon is most pronounced in animals that undergo a postembryonic metamorphosis such as amphibians (Kimmel and Model, 1978) and insects (Taylor and Truman, 1974) but it also occurs during embryonic development (e.g., Goodman and Bate, 1981).

The past few years have seen a surge of interest in the role of neuronal death in the development and function of the nervous systems of various invertebrates (Goodman and Bate, 1981; Horvitz et al., 1982). In these cases the ability to follow the origins and fates of identified cells has provided insights into a number of problems

\footnotetext{
${ }^{1}$ We thank Prof. L. M. Riddiford for a critical reading of the manuscript and Dr. S. R. Reiss for preparing the histological material The research was supported through grants from the National Institutes of Health (NS 13079), the National Science Foundation (PCM 80-20975), and the McKnight Foundation.

${ }^{2}$ Department of Physiology and Biophysics, University of Washington, Seattle, WA 98195.
}

including the importance of cellular lineage in determining the fate of cells. Although lineage is clearly a key in understanding the fate of a particular cell, it is not known whether extrinsic factors such as hormones play a role in actually triggering the degeneration.

The first paper in this series (Truman, 1983) described the pattern of neuronal degeneration in newly emerged adults of the moth, Manduca sexta. This species shows the death of approximately $50 \%$ of its abdominal motoneurons during the first 2 days of adult life (Taylor and Truman, 1974). The cells that are lost are ones that control the behavior of the animal through metamorphosis, but after emergence these circuits are apparently no longer needed and hence degenerate. The death of these cells occurs according to a precise temporal program in which each cell has its own characteristic time to die relative to the time of emergence of the moth. The present paper examines the control over the onset of this program of degeneration and presents evidence that it is regulated by levels of circulating steroids.

\section{Materials and Methods}

The tobacco hornworms, $M$. sexta, were reared individually on an artificial diet as described previously (Truman, 1983). Developing adults were exposed to a 12-hour light, 12-hour dark photoperiod with a superimposed $27^{\circ} \mathrm{C}-25^{\circ} \mathrm{C}$ temperature cycle to improve the synchrony of adult ecdysis (Lockshin et al., 1975). For all designa- 
tions of times in this paper, lights-off is arbitrarily designated as midnight (12 P.M.).

Adult development in Manduca requires an average of 18 days at $26^{\circ} \mathrm{C}$. During the last third of this period, the exact stage of development can be determined by features of the forming moth that are visible through the old pupal cuticle. The characteristics of these last days of development are given by Schwartz and Truman (1983). By the 18th day of development the insects had formed a new pigmented adult cuticle, the inner layers of the surrounding pupal cuticle were digested, and the moths emerged (ecdysed) from the old cuticle at 10 P.M. (range of $\pm 1.5 \mathrm{hr}$ ). Newly emerged moths were placed in paper bags and held at $26^{\circ} \mathrm{C}$ until needed.

Surgery was performed on Manduca that were in the second day of adult development. Animals were anesthetized with $\mathrm{CO}_{2}$, and tissues were implanted through an incision in the abdominal cuticle. A few crystals of phenylthiourea were added to the wound to inhibit tyrosinase activity (Williams, 1959), and the wound was then covered by a fragment of plastic coverslip that was sealed to the cuticle with molten wax.

Crystalline 20-hydroxyecdysone (20-HE) was obtained from Sigma Chemical Co. Solutions were made in saline (Ephrussi and Beadle, 1936), and the concentration was checked spectrophotometrically at $240 \mathrm{~nm}$ with a molar extinction coefficient of 12,670 (Meltzer, 1971). Solutions were injected into the dorsum of the thorax by means of a Hamilton syringe. For infusions the syringes were connected to the animal through a length of PE-10 tubing (Clay-Adams) that was implanted into the thoracic heart and waxed to the overlying cuticle (Reynolds et al., 1979). The plungers of the syringes were automatically advanced by a Sage syringe pump.

At appropriate times the abdomen was severed from the insect, eviscerated, and flooded with alcoholic Bouin's fixative for at least $2 \mathrm{hr}$. The fourth abdominal ganglion (A4) was removed, dehydrated, and embedded in paraffin using standard techniques. Ganglia were serially sectioned at $10 \mu \mathrm{m}$, and the sections were stained with hematoxylin and eosin. Preparations were examined at $\times 400$ through a Leitz Ortholux II microscope. Degenerating neurons appeared as condensed, darkly staining bodies without a distinct nucleus. The neurons fell into two size classes: small cells with cell body diameters from 7 to $15 \mu \mathrm{m}$ (almost exclusively interneurons); and large cells with somatas from 20 to $40 \mu \mathrm{m}$ (primarily motoneurons). The size distinction between the two groups was evident through most of their degeneration so that counts of dying cells could be made separately for the two groups (Truman, 1983).

The unique position of the cell body of a number of the motoneurons allowed these cells to be repeatedly identified in serially sectioned material; hence, they could be followed as individuals through their degeneration. The progression of breakdown of these cells was divided into five sequential stages ('Truman, 1983): Stage 0 cells were healthy cells showing a round or oval nucleus surrounded by moderately staining cytoplasm; stage 1 cells had an irregular nuclear outline and pale cytoplasm; stage 2 was characterized by a collapsed, darkly staining nucleus; stage 3 showed the entire cell body to be con- densed and darkly staining; and in stage 4 the degenerating soma was reduced in size and the staining was very pale. A given motoneuron required about $60 \mathrm{hr}$ to progress through these stages.

In most cases the state of neuronal degeneration in ganglion A4 was quantified by monitoring the fate of the three pairs of motoneurons that comprise the D-IV group. These neurons innervate the ventral set of intersegmental muscles (R. B. Levine and J. W. Truman, unpublished observations), and their somata are located in the anterodorsomedial region of the ganglion.

\section{Results}

\section{Neuronal death in implanted ganglia}

The chain of ventral ganglia from A3 to A8 was removed from Manduca on the second day of adult development and implanted into the dorsal region of segment A3 in animals of the same age. About 3 weeks later, around the time of adult ecdysis, the implant was recovered and the state of neuronal degeneration was compared to that seen in the nervous system of the host. The shape and dimensions of the implant were often distorted after the completion of metamorphosis, so it was not possible to identify unambiguously particular neurons on the basis of cell body location alone. Consequently, we compared the total number of degenerating motoneurons (somata diameter, $>20 \mu \mathrm{m}$ ) and interneurons (somata diameter, $<15 \mu \mathrm{m}$ ) in implant and host ganglia.

As seen in Figure 1, implants taken from Manduca immediately prior to ecdysis showed no degenerating motoneurons and few dying interneurons. By contrast those removed at 20 to $40 \mathrm{hr}$ after ecdysis contained dying cells from both classes. Importantly, the progression of neuronal death in the implants paralleled that observed in the CNS of their host. This was clearly seen in the case of the interneurons and also appeared true for the motoneurons except, for the latter, the total number of cells dying in the implant was consistently less than that seen in host ganglia.

In these experiments the trauma of the implantation surgery delayed the development of the hosts to a varia-

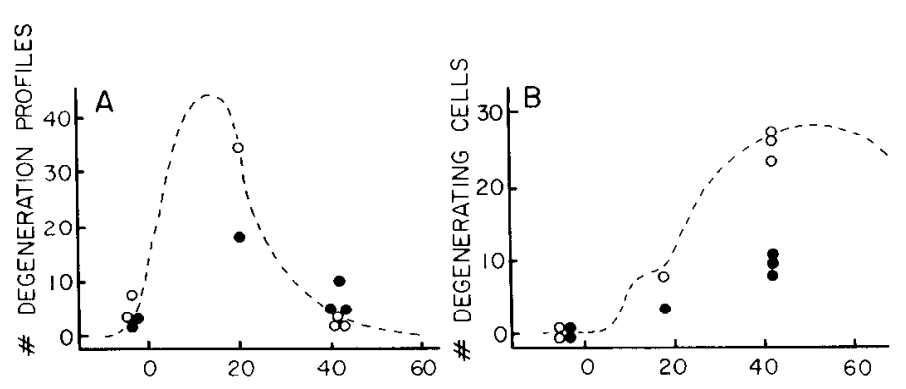

Figure 1. Neuronal degeneration in ganglia implanted into the abdomen of Manduca. Total numbers of degenerating interneurons $(A)$ and motoneurons $(B)$ in sectioned $\mathrm{A} 4$ ganglia of host $(O)$ and in the sectioned CNS implant (O). Interneuron counts are given as "profiles" because the same cell might be counted in adjacent sections; each dying motoneuron was counted only once. - - - , normal appearance of degenerating cells in A4 from control animals as published by Truman (1983). 
ble degree such that they completed this process from 21 to 24 days after the operation. Implants were recovered from the first animals to finish development at 1 or 2 days post-ecdysis, whereas those taken from the slower hosts were recovered at or before ecdysis. Importantly, the state of cell death in the implant was not related to the absolute age of the implant but was a function only of the developmental condition of the host. Thus, the cell death does not appear to be ganglion autonomous but rather is coordinated with the host's development. Since neural connections with the CNS of the host are not required for this coordination, it most probably must be established through a blood-borne factor.

It was of interest that the implants showed fewer dying motoneurons than did host ganglia (Fig. 1). Counts of the total number of healthy neurons in the implants also showed fewer cells than did the ganglia of the hosts, indicating that some neurons did not survive transplantation and the subsequent metamorphosis in their ectopic location. This reduced survival rate could be due to a number of factors including a lack of appropriate peripheral targets or necessary descending inputs. Whatever these factors may be, their effects are probably manifest early in metamorphosis since there are few signs of death in the implants immediately before adult ecdysis. Thus, among the transplanted neurons that survive through metamorphosis, some then later die in response to some signal that occurs around the time of ecdysis.

\section{Effects of abdomen isolation on cell degeneration}

The abdominal intersegmental muscles of Manduca die soon after adult ecdysis, and this death is triggered by a decline in the levels of circulating ecdysteroids at the end of adult development (Schwartz and Truman, 1983). Abdomens could be isolated from the centers that produce ecdysteroids (the prothoracic glands in the thorax) by the simple procedure of clamping a hemostat between the thorax and abdomen and discarding the anterior piece. These isolated abdomens subsequently live for a number of weeks. We isolated abdomens at various times during the last 3 days of adult development and assessed the state of degeneration within the CNS at $16 \mathrm{hr}$ after the ecdysis of controls. The condition of the D-IV motoneurons was used as a quantitative marker of the progression of the neuronal degeneration (see "Materials and Methods"). When abdomens were isolated on days 17 or 18 of adult development, the D-IV neurons subsequently showed the same state of degeneration as did controls (Table I). By contrast abdominal isolation on days 15 or 16 resulted in precocious neuronal death; the average degeneration stage of the neurons in these preparations indicated an advancement of about $24 \mathrm{hr}$ in the time of cell death.

The early death shown by the D-IV motoneurons after abdominal isolation was also shared by other neurons within the ganglion. Figure 2 follows the time course of degeneration of selected identified motoneurons in abdomens that had been isolated on day 16 . The sequence of motoneuron death in the isolated abdomens (MN-11, then the D-IV cells, MN-2, and MN-12) was the same as seen in intact animals. The first three sets of moto-
TABLE I

Effects of the time of abdomen isolation on the subsequent state of degeneration of the D-IV motoneurons in ganglion A4

$\begin{array}{ccc}\text { Time of Isolation }^{\alpha} & N & {\text { Degeneration Stage }(\text { mean } \pm \mathrm{SD})^{b}}^{\mathrm{b}} \\ \text { Intact } & 20 & 0.6 \pm 0.5 \\ \text { Day 18 } & 13 & 0.3 \pm 0.4 \\ \text { Day 17 } & 10 & 0.5 \pm 0.4 \\ \text { Day 16 } & 24 & 1.8 \pm 0.5 \\ \text { Day 15 } & 13 & 2.1 \pm 1.0\end{array}$

${ }^{a}$ Abdominal isolations were at 6 P.M. on the indicated day of development.

${ }^{b}$ Ganglia were examined at $16 \mathrm{hr}$ after the ecdysis of control Manduca.
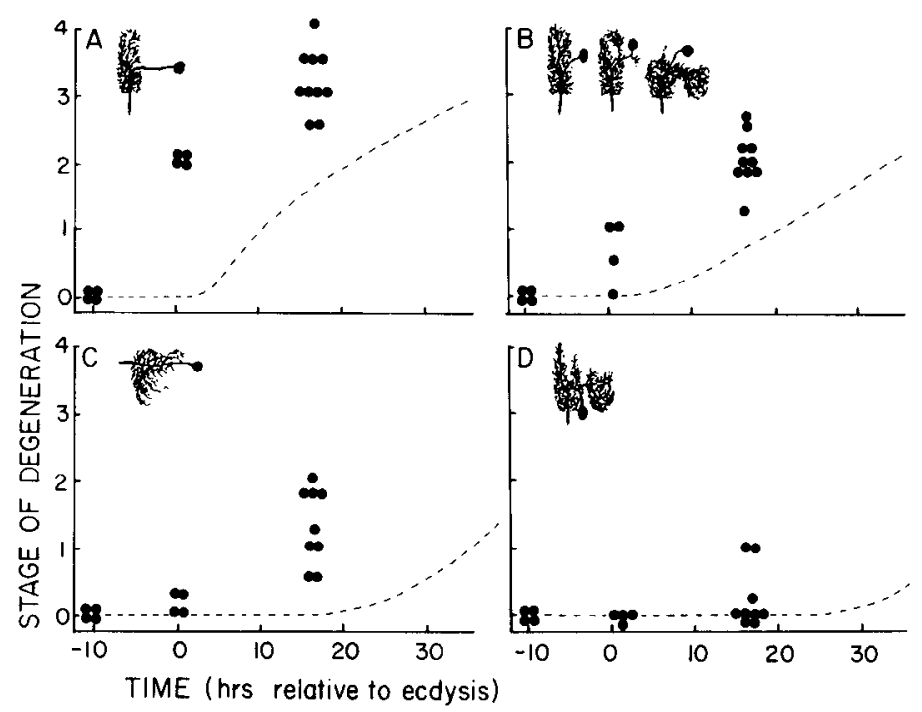

Figure 2. Time course of the degeneration of specific identified motoneurons in abdomens that were isolated on day 16 of adult development. $A, \mathrm{MN}-11 ; B$, the D-IV group; $C, \mathrm{MN}-2 ; D$, MN-12. The neurons were examined at various times relative to the ecdysis of control moths. Each point represents the average stage of degeneration of the respective neurons in a single abdominal ganglion. Insets, central morphology of the cells. - - - , normal time course of degeneration of the respective cells in control insects as described by Truman (1983).

neurons showed a clear advancement in the time of death; MN-12 was probably also advanced but later time points need to be taken in order to establish this point conclusively. Thus, after abdominal isolation the characteristic order of cell death is maintained but the whole temporal program is advanced by about $24 \mathrm{hr}$.

Although the temporal pattern of cell death was unaffected by this manipulation, abdominal isolation did slightly disrupt the coordination of degeneration within a group. For example, the three pairs of neurons that comprise the D-IV group normally die in relative synchrony; in any given ganglion the variation between the most extreme cells in the group is at most two degeneration stages ( $\leq 10 \mathrm{hr}$ ). In isolated abdomens, by contrast, the variance between the cells of that group was much greater (Table II), and we sometimes found intact (stage 0) D-IV neurons in the same ganglion with other D-IV cells that were almost completely degenerated (stage 4). 
The latter situation represents a difference of at least 30 $\mathrm{hr}$ in degeneration times.

Abdominal isolation could result in premature neuronal death either by disrupting neural connections with anterior centers or by interrupting the flow of an endocrine factor from the thorax to the abdomen. Data from two experiments favor the latter hypothesis. In the first set of experiments the ventral connectives were transected just caudal to the thoracic juncture in day 15 Manduca. At $16 \mathrm{hr}$ after the attempted ecdysis of these insects, the D-IV neurons in ganglion A4 showed an average degeneration score of $0.5 \pm 0.4(\mathrm{SD}, N=8)$ as compared with 0.6 for unoperated controls and 2.1 for abdomens isolated on day 15 (Table I). Thus, the surgical transection of the nerve cord in an otherwise intact insect does not cause early neuron degeneration.

Results implicating the prothoracic glands as the source of a hormone that regulated the time of cell death came from experiments in which pairs of these glands were removed from day 2 developing adults and implanted into the abdomens of Manduca of the same stage. When these insects subsequently attained day 15 or 16 of development, each was then paired with a control insect that was judged to be at the same stage of development based on its cuticular markers. The abdomens of both were isolated and the nervous systems examined 3 to 4 days later to determine the state of D-IV neuronal degeneration. In 6 of 7 pairs the control abdomen showed advanced degencration over that seen in the abdomen with the gland implant. The D-IV neurons in the former showed an average degeneration score of $2.9 \pm 1.0$ whereas the latter had a score of only $1.2 \pm 1.3$. The difference is statistically significant ( $p<0.02, t$ test) and represents a difference between the two groups of about $20 \mathrm{hr}$ in the time of onset of degeneration. Thus, the presence of a prothoracic gland is sufficient to prevent the precocious neuronal degeneration seen after abdomen isolation.

\section{Ecdysteroid treatments and the timing of neuronal death}

The above experiments clearly implicate the ecdysteroids (the products of the prothoracic glands) in the regulation of neuronal degeneration. Abdominal isolation on day 15 or 16 causes a precocious drop in the circulating ecdysteroid titer (Schwartz and Truman, 1983), which is correlated with the early death of the neurons. Consequently, it appears that the neurons become steroid dependent during adult development and that the decline in the ecdysteroid titer that occurs at the end of metamorphosis results in cell death. This hypothesis was

TABLE II

Effects of abdominal isolation on the variability in the state of degeneration of motoneurons in the D-IV group

\begin{tabular}{|c|c|c|c|c|c|c|}
\hline \multirow[t]{2}{*}{ Treatment } & \multirow[t]{2}{*}{$N$} & \multicolumn{5}{|c|}{$\begin{array}{l}\% \text { of Ganglia in Which } \\
\text { Members of Group } \\
\text { Spanned Indicated No. of } \\
\text { Categories }\end{array}$} \\
\hline & & 1 & 2 & 3 & 4 & $\overline{5}$ \\
\hline None & 86 & 13 & 45 & 41 & 1 & 0 \\
\hline Isolated abdomen & 54 & 9 & 6 & 44 & 33 & 7 \\
\hline
\end{tabular}

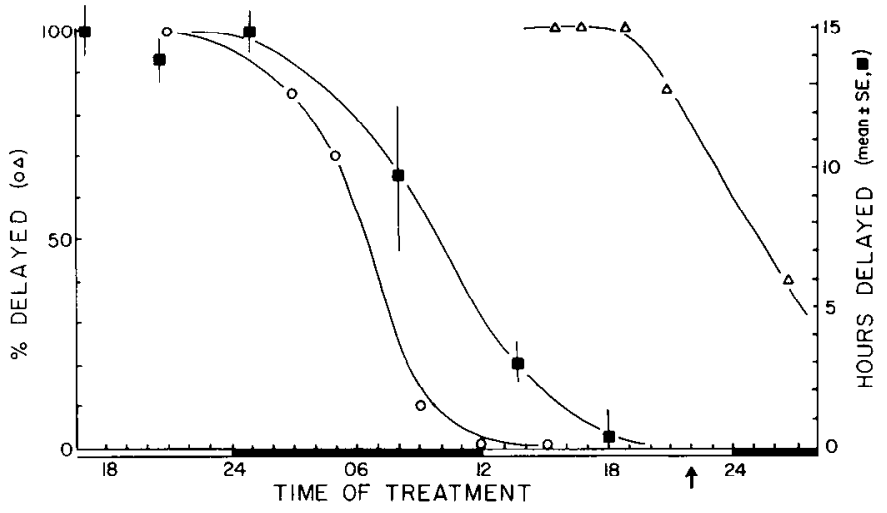

Figure 3. Effects of the time of ecdysteroid administration on the ability of the treatment to delay ecdysis behavior $(O)$, intersegmental muscle degeneration $(\mathbb{G})$, and D-IV motoneuron degeneration $(\triangle)$. Insects were injected with $5 \mu \mathrm{g} 20-\mathrm{HE} / \mathrm{gm}$ at the times indicated. Black and white bars, nights and days, respectively, of the last $32 \mathrm{hr}$ of adult development and the first $6 \mathrm{hr}$ of adult life. Ecdysis and motoneuron data based on 10 insects/point, muscle data based on 5 to 6 insects/point. Arrow, normal time of adult ecdysis.

initially tested by artificially maintaining high blood ecdysteroid levels at the end of development. Catheters were implanted into the hearts of day $17 \mathrm{Manduca}$, and the insects were continuously infused with ecdysteroids at the rate of $70 \mathrm{ng}$ of $20-\mathrm{HE} / \mathrm{gm} / \mathrm{hr}$. Eleven insects were infused in this manner for periods ranging from 2 to 7 days. In all cases, at the end of the treatment the D-IV neurons were still alive as were the other motoneurons and interneurons that normally should have died. Thus, maintenance of high ecdysteroid levels late in adult development completely prevents the neuronal degeneration.

Interpretation of the above infusion experiments is complicated by the fact that steroid treatment late in adult development inhibits a number of processes including the death of the intersegmental muscles (Schwartz. and Truman, 1983) and adult ecdysis behavior itself (Truman et al., 1983). Thus, the effect of this treatment on the neuronal death may be an indirect result of the action of the steroid on some other tissue. Evidence for a direct action of the steroid on the CNS was obtained by giving single injections of 20-HE. A dosage of $5 \mu \mathrm{g} /$ gm delayed all of these various processes, but the effectiveness of the treatment was strictly dependent on the time of application. When given at progressively later times through day 17 and the night of day 18, the effectiveness of the injections declined such that by lights-on of day 18 the steroid treatment no longer blocked ecdysis behavior (Fig. 3; Truman et al., 1983). The intersegmental muscles became insensitive to 20 HE treatment shortly thereafter (Fig. 3; Schwartz and Truman, 1983). By contrast the D-IV neurons remained sensitive for about 14 more $\mathrm{hr}$, and their death could still be prevented by $20-\mathrm{HE}$ given at the time of adult ecdysis (Fig. 3). Thus, by varying the time of 20 -HE treatment it was possible to dissociate motoneuron degeneration from ecdysis behavior and from the death of their target muscles. This suggests that the postecdysial death of the 
D-IV neurons is not rigidly coupled to the other known physiological changes at this time and probably represents a direct response of the CNS to the declining steroid levels.

The time of onset of neuronal degeneration can be delayed by relatively low levels of 20 -HE. Figure 4 shows the response of Manduca to a range of ecdysteroid dosages administered at 2 P.M. on day 18 . The extent of the delay in degeneration, as typified by the score of the DIV cells, was proportional to the dosage of $20-\mathrm{HE}$ injected. Delays were seen down to the lowest level of ecdysteroid tested which was $125 \mathrm{ng} / \mathrm{gm}$. These levels are in the range of those determined by radioimmunoassay in whole-body extracts of Manduca late in adult development (Bollenhacher et al., 1981).

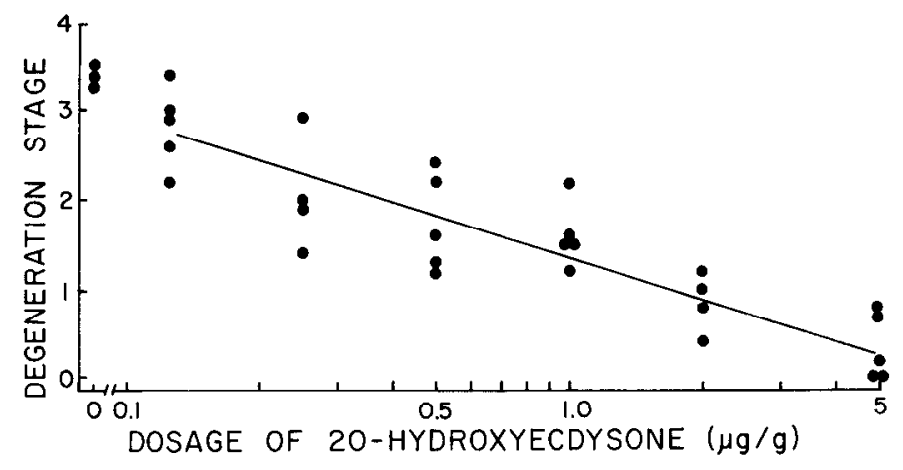

Figure 4. Effects of dosage of 20 -HE on the subsequent degeneration of the D-IV motoneurons. Insects were treated at 2 P.M. on day 18 of adult development, and the neurons were then scored 2 days after the subsequent ecdysis. Each point represents the average score of the D-IV neurons from one animal,

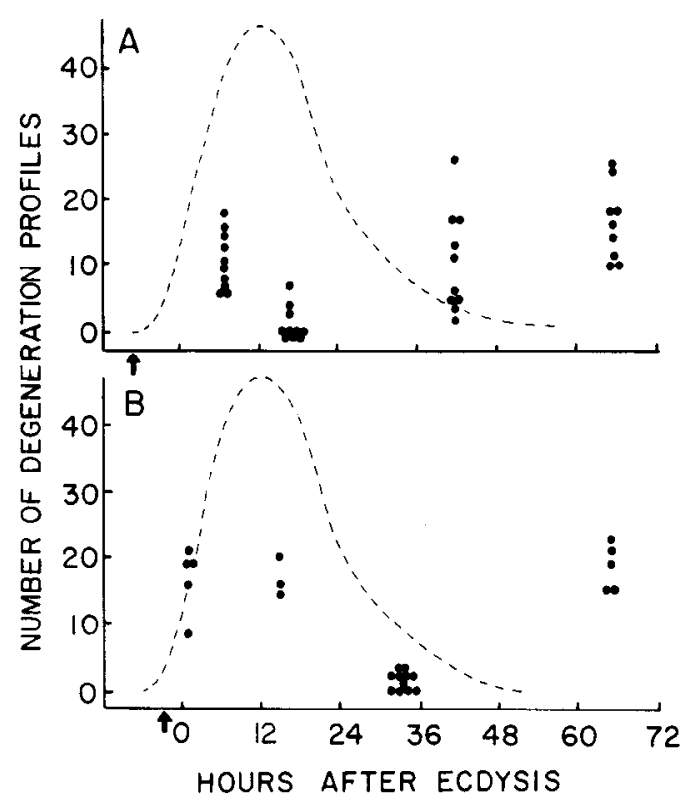

Figure 5. Effect of ecdysteroid injection ( $5 \mu \mathrm{g} 20-\mathrm{HE} / \mathrm{gm}$ on the time course of degeneration of interneurons in ganglion A4. Injections (arrow) were before ecdysis at $(A)$ 3:30 P.M. or $(B)$ 7 P.M. on day 18. Each point represents counts from a single ganglion. - - - -, time course of neuronal death in untreated controls.

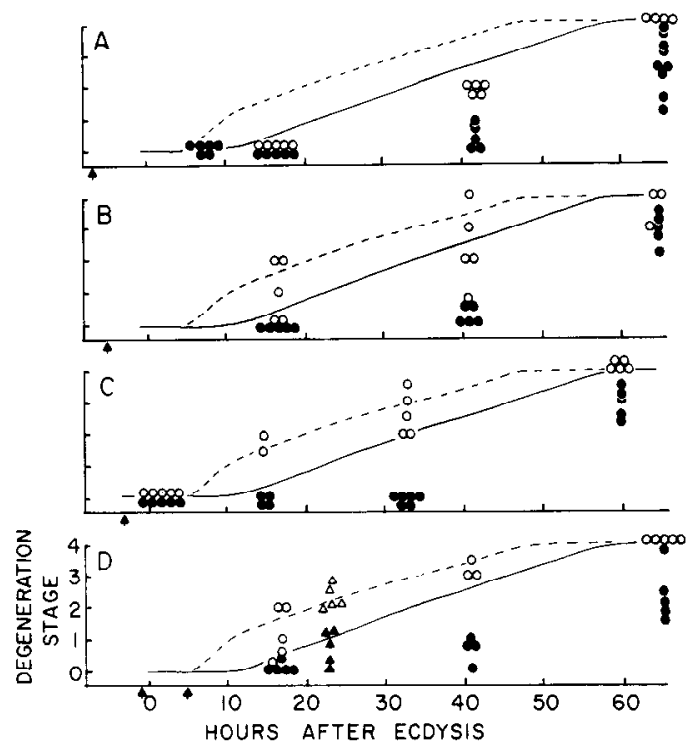

Figure 6. Effect of the time of ecdysteroid treatment (5 $\mu \mathrm{g}$ $20-\mathrm{HE} / \mathrm{gm}$ ) on the course of degeneration of the pair of $\mathrm{MN}$ 11 cells $(O, \triangle)$ and the $D$-IV neurons $(\boldsymbol{O}, \mathbf{\Delta})$. Injections (arrows) were on the last day of adult development at $(A)$ 3:30 P.M., (B) 5 P.M., (C) 7 P.M., (D) 9 P.M. $(\mathrm{O}, \ominus)$, or 3 A.M. $(\triangle, \Delta)$. In the case of the last group, the insects were injected as newly ecdysed moths. Each point represents the state of the cells in a single ganglion. __ , - - - , normal progression of degeneration for MN-11 and the D-IV cells, respectively.

\section{Ecdysteroid disruption of the temporal program of neuronal death}

In most of the studies reported here, the state of the D-IV neurons was a reliable marker for the fate of the other neurons within ganglion A4. However, this was not the case when ecdysteroids were injected very late in development. Such treatments prevented the death of some cells while allowing others to die on schedule. Figure 5 follows the time course of interneuron degeneration in Manduca that were injected with $5 \mu \mathrm{g}$ of 20 HE/gm at 3:30 P.M. or 7 P.M. on day 18. In both cases interneuron death appeared to begin on time, only to be interrupted partway through the program. During this interruption cells already under way in degeneration finished the process, but no new cells began to break down until after a substantial delay. Importantly, the later injection allowed more cells to die before the interruption than did the earlier one.

The impact of the timing of ecdysteroid treatment on the death of individual cells is more clearly seen for the motoneurons. The response of MN-11 and the D-IV cells to ecdysteroids administered at various times is summarized in Figure 6. Ecdysteroid treatment at 3:30 P.M. on day 18 delayed the death of both cell groups. Treatment at 5 P.M. delayed some of the MN-11 cells, but others showed a time course of degeneration similar to that of untreated insects. By contrast all of the D-IV cells in these ganglia were retarded in the time of their degeneration. Two hours later the same treatment continued to delay the death of the D-IV group, while all of the MN-11 cells died on schedule. The results were similar at 9 P.M. except that a few of the D-IV cells 


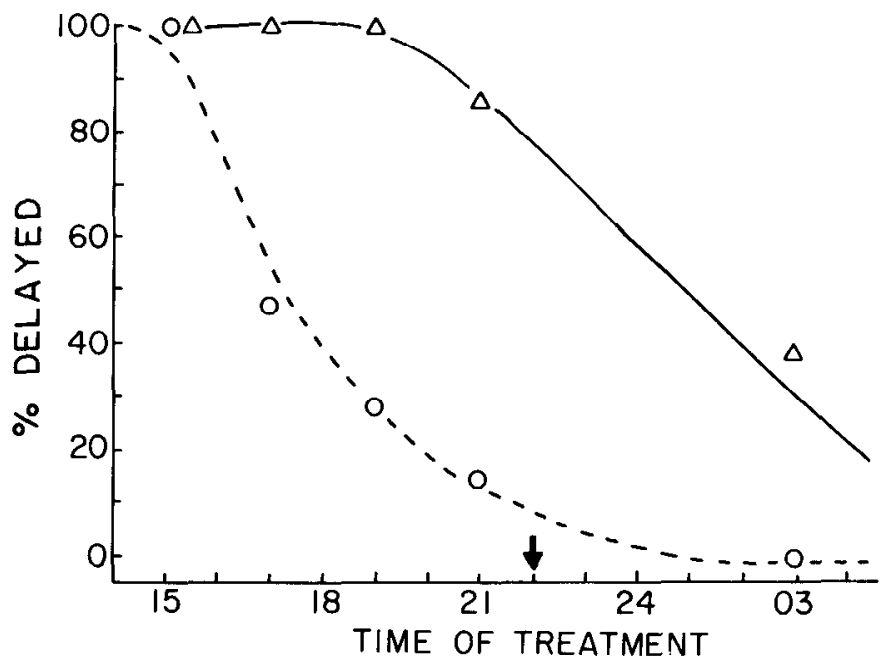

Figure 7. Relationship of the time of ecdysteroid treatment to the ability of the injection to delay the degeneration of $M N$ $11(\mathrm{O})$ and the D-IV motoneurons $(\triangle)$. Neurons were considered delayed if their stage of degeneration was more than 2 SD less than that seen for the state of the respective neurons in intact control moths. Arrow, normal time of adult ecdysis.

begin to show a normal time of death. By $6 \mathrm{hr}$ later most of the D-IV cells were committed to die and could no longer be saved by the steroid. These results indicate that for each set of neurons one can identify a critical period, before which ecdysteroid treatment will delay death but after which the cells will degenerate on schedule even if steroid is provided (Fig. 7). The critical periods for different cells vary, and for the D-IV and MN-11 cells they are separated by about $8 \mathrm{hr}$.

\section{Discussion}

Hormones have an important role in regulating neuronal death in a number of systems. In the case of metamorphosis of some amphibians, thyroxine has been implicated in the degeneration of the Mauthner cells (Kimmel and Model, 1978; Pesetsky, 1966). Similarly, sex steroids have been shown to regulate the sex-specific cell death that occurs in a spinal nucleus in the rat (Jordan et al., 1982). The data presented above show that hormones can also control cell death in the nervous system of an invertebrate. In Manduca the transformation of the pupal stage into the adult moth is initiated by the appearance of ecdysteroids (Riddiford, 1980). The subsequent decline in steroid levels that occurs at the end of metamorphosis serves as the signal for the degeneration of about half of the abdominal motoneurons and interneurons. Manipulations, such as abdominal isolation, that cause a precocious decline in the ecdysteroid titer also result in an advancement in the time of death of the cells. Treatments that augment the ecdysteroid titer (infusion or injections of 20 -HE or implantation of prothoracic glands) prolong the life of the cells.

The stereotyped program of neuronal degeneration is temporally associated with ecdysis behavior of the adult. However, cell death is not triggered by the performance of this behavior. Abdomens isolated on day 18 do not undergo ecdysis movements, but they nevertheless show a normal program of neuronal degeneration. Also, as shown in Figure 4, properly timed injections of $20-\mathrm{HE}$ block cell death in animals that show a normal ecdysis behavior. Similar experiments also show that death of a motoneuron is not triggered by the degeneration of its target muscle. Most probably the CNS is responding directly to the steroid decline. A major question remains, however, as to whether each cell that dies does so as a direct response to the steroid titer or whether there are only a few target cells and other cells die as a result of the death of these few. The latter hypothesis is hard to reconcile with the results of treating Manduca with ecdysteroids at various times during the last day of development. These experiments indicate that the death of a particular neuron does not follow irrevocably from the death of the neurons that died earlier. Another pertinent observation comes from examining the response of $\mathrm{MN}$ 11 to hormone treatment during its critical period. Occasionally, we found a neuron that was degenerating on schedule in the same ganglion with its contralateral homologue that was delayed by the treatment. This result is most easily explained by the hypothesis that each cell responds directly to the ecdysteroid treatment. Obviously, a definitive test of this hypothesis will require a study of the response of individual cultured neurons to the presence or absence of ecdysteroids.

An intriguing aspect of cell death in Manduca is the precise temporal order in which the cells die. This ordering of the times of degeneration is apparently set prior to the middle of the photophase on day 17 since abdominal isolation at this time or later results in normal degeneration. Importantly, although abdominal isolation at earlier times results in precocious degeneration, the order of cell death after this treatment remains normal (Fig. 2). The mechanism for the ordering of the time of cell death is not known.

It is of interest that the order of cell death is also reflected by the order in which each neuron becomes insensitive to ecdysteroids. In the case of both MN-11 and the D-IV group, the cells become insensitive to ecdysteroid treatment (Fig. 7) about $12 \mathrm{hr}$ before they show the first ultrastructural (Stocker et al., 1978) and cytological (Truman, 1983) changes characteristic of degeneration. Presumably, the critical period represents the time that the cell initiates a set of biochemical processes which will eventually lead to its dissolution. Up until the time that these processes have started, they apparently can be inhibited by steroid, but after they have begun, ecdysteroids have no effect on the subsequent course of events. The nature of these biochemical events in the moth nervous system is unknown, but the hormonally triggered death of the intersegmental muscles in these moths appears to require the production of new mRNAs and proteins (Lockshin, 1969). Also, in the nematode, Caenorhabdites elegans, the programmed degeneration of cells in the nervous system appears to require the production of a particular gene product (Horvitz et al., 1982). If such is the case for the nervous system of Manduca and if steroids are acting at the level of transcription to control neuronal death, then approximately $12 \mathrm{hr}$ elapse between the transcription of this gene and the first visible sign of cell death. 
The motoneurons that degenerate in the adult were born during embryonic development. There are times during the postembryonic life of the animal when these cells are exposed to an environment that is devoid of ecdysteroids. The most notable time is just after pupal ecdysis when, depending on environmental conditions, the insect may enter a state of developmental arrest (diapause). The diapause state is maintained by the lack of ecdysteroids, and yet these neurons survive for at least several months without ill effect (J. W. Truman, unpublished observations). Consequently, at this stage they do not require ecdysteroids in order to survive. The subsequent appearance of ecdysteroids that terminates diapause and promotes adult development presumably then renders these cells dependent on ecdysteroids for their subsequent survival. The nature of the changes that might occur in these cells to make them steroid dependent is unknown.

\section{References}

Bollenbacher, W. E., S. L. Smith, W. Goodman, and L. I. Gilbert (1981) Ecdysteroid titer during larval-pupal-adult development of the tobacco hornworm, Manduca sexta. Gen. Comp. Endocrinol. 44: 302-306.

Ephrussi, B., and G. W. Beadle (1936) A technique of transplantation for Drosophila. Am. Nat. 70: 218-225.

Goodman, C. S., and M. Bate (1981) Neuronal development in the grasshopper. Trends Neurosci. 4: 163-169.

Hamburger, V., and R. W. Oppenheim (1982) Naturally occurring neuronal death in vertebrates. Neurosci. Commentaries 1: $39-55$.

Horvitz, H. R., H. M. Ellis, and P. W. Sternberg (1982) Programmed cell death in nematode development. Neurosci. Commentaries 1: 56-65.

Jordan, C. L., S. M. Breedlove, and A. P. Arnold (1982) Neurogenesis of motoneurons in the sexually dimorphic spinal nucleus of the bulbocavernosus in rats. Soc. Neurosci. Abstr. 8: 198.
Kimmel, C. B., and P. Model (1978) Developmental studies of the Mauthner cell. In Neurobiology of the Mauthner Cell, D. S. Faber and H. Korn, eds., pp. 183-220, Raven Press, New York.

Lockshin, R. A. (1969) Programmed cell death. Activation of lysis by a mechanism involving the synthesis of protein. J. Insect Physiol. 15: 1505-1516.

Lockshin, R. A., M. Rosett, and K. Srokose (1975) Control of ecdysis by heat in Manduca sexta. I. Insect Physiol. 21: 17991802.

Meltzer, Y. L. (1971) Hormonal and Attractant Pesticide Technology. Noyes Data Corp., Park Ridge, NJ.

Pesetsky, I. (1966) The role of the thyroid in the development of the Mauthner's neuron. A karyometric study in thyroidectomized larvae. Z. Zellforsch. Mikrosk. Anat. 75: 138-145.

Reynolds, S. E., P. H. Taghert, and J. W. Truman (1979) Eclosion hormone and bursicon titres and the onset of hormonal responsiveness during the last day of adult development in Manduca sexta (L.). J. Exp. Biol. 78: 77-86.

Riddiford, L. M. (1980) Insect endocrinology: Action of hormones at the cellular level. Annu. Rev. Physiol. 42: 511-528.

Schwartz, L. M., and J. W. Truman (1983) Hormonal control of rates of metamorphic development in the tobacco hornworm Manduca sexta. Dev. Biol. 99: 103-114.

Stocker, R. F., J. S. Edwards, and J. W. Truman (1978) Fine structure of degenerating moth abdominal motor neurons after eclosion. Cell Tissue Res. 191: 317-331.

Taylor, H. M., and J. W. Truman (1974) Metamorphosis of the abdominal ganglia of the tobacco hornworm, Manduca sexta: Changes in populations of identified motor neurons. J. Comp. Physiol. 90: 367-388.

Truman, J. W. (1983) Programmed cell death in the nervous system of an adult insect. J. Comp. Neurol. 216: 445-452.

Truman, J. W., D. B. Rountree, S. E. Reiss, and L. M. Schwartz (1983) Ecdysteroids regulate the release and action of eclosion hormone in the tobacco hornworm, Manduca sexta. J. Insect Physiol., in press.

Williams, C. M. (1959) The juvenile hormone. I. Endocrine activity of the corpora allata of the adult Cecropia silkworm. Biol. Bull. 116: 323-338. 